\title{
Standardisation and harmonisation boost the credibility of nuclear medicine procedures
}

\author{
Klaus Tatsch
}

Published online: 17 November 2011

(C) Springer-Verlag 2011

The results of diagnostic procedures in nuclear medicine may be heavily influenced by the equipment used and factors related to the investigator. The freedom of physicists, technicians and physicians to discretely compose the camera set-up and the protocols for acquisition, reconstruction and post-processing, including various techniques of quantification, not surprisingly results in a heterogeneous outcome of procedures that are in principle the same. One might debate whether these issues play a role in the diagnostic work-up of a certain patient; it is quite evident, however, that scientific investigations, e.g. in the setting of multicentre trials, may be greatly compromised by these factors.

Procedural guidelines published by professional associations in our field, such as the European Association of Nuclear Medicine (EANM), the Society of Nuclear Medicine (SNM) or the local societies in various European countries are an important milestone to provide a basic framework. However, it is in the nature of such guidelines that they leave space for interpretation and layout. Particularly for scientific purposes but also in the clinical context standardisation and harmonisation of procedures needs to be further pursued and developed to improve the comparability of results between institutions. The credibility of reported findings depends largely on their validity. The latter is a crucial point, because nuclear medicine procedures increasingly have to prove

K. Tatsch $(\square)$

Klinik für Nuklearmedizin, Städtisches Klinikum Karlsruhe,

Moltkestr. 90,

76133 Karlsruhe, Germany

e-mail: Klaus.Tatsch@klinikum-karlsruhe.de

K. Tatsch

EANM Research Ltd.,

Hollandstrasse 14/Mezzanine,

1020 Vienna, Austria both benefit for the patient and economic benefit before they are accepted and recognized by health insurance and authorities.

In this context procedural guidelines have to be further narrowed with the aim of providing harmonised, wellaccepted results. An example of how these theoretical considerations can be successfully realised is supplied by John Dickson and co-workers. They report in this issue of the European Journal of Nuclear Medicine and Molecular Imaging a proposal for the standardisation of SPECT investigations of the brain for multicentre trials [1]. This proposal is the first logical step of a remarkable programme launched and developed under the umbrella of the recently founded EANM Research Ltd (EARL) and aims to standardise investigations with I-123-FP-CIT (DaTSCAN) SPECT in Europe. FP-CIT is a diagnostically valuable tracer for assessment of presynaptic dopaminergic function, particularly in neurodegenerative Parkinsonian syndromes. Unfortunately however, even though this radiopharmaceutical has been approved for more than a decade in Europe in clinical practice as well as in clinical trials, the quality spectrum of scans has ranged from excellent to rather poor, mostly due to the lack of standardised acquisition, processing, and quantification and of a generally available reference database. Realising these problems, the Neuroimaging Committee of the EANM decided to initiate a programme to overcome these limitations by setting up an investigatorinitiated multicentre trial using EARL as a platform. The aims of the trial, performed at 15 highly experienced centres in ten European countries, were threefold: first, to provide a framework to acquire high-quality multicentre SPECT studies by proposing a standardised set-up and qualityassurance regime; second, to perform anthropomorphic phantom measurements for cross-calibration of gamma camera systems; and third, to build up a comprehensive 
reference database of healthy controls. The paper by John Dickson and co-workers deals with the first aim. Their proposal describes a framework for performing high-quality multicentre trials with SPECT using a pan-European initiative to acquire a normal control dopamine transporter brain scan database as an example. In this initiative a total of 24 gamma cameras from 8 different manufacturers at 15 different sites have been evaluated - to my knowledge one of the largest comparative series published. Three topics were addressed: quality assurance of the imaging device, standardisation of imaging protocols, and system characterisation. Quality assurance covered parameters such as intrinsic and system uniformity, centre of rotation, pixel size of SPECT acquisitions and full width at half maximum SPECT resolution. Imaging protocol parameters based on EANM guidelines have been suggested, for instance, for acquisition parameters, including minimum number of total counts, number of projections, pixel size, energy windows, collimators and reconstruction details [2]. System characterisation with anthropomorphic phantom measurements has been extended and reported by a further publication in the meantime and has demonstrated that camera-specific recovery coefficients could be derived from linear regression analysis [3]. Based on these two prerequisites, harmonisation of results and direct comparison of patient investigations between centres should become possible. The third step of the project, to establish a database of FP-CIT SPECT scans of healthy controls strictly following the mentioned standardising procedures, has also now been concluded, including about 150 healthy subjects covering the age range between 20 and 90 years. A manuscript presenting an extensive analysis of these data is currently in preparation. Thus, the final goal - to provide a highly standardised European database allowing correction of quantitative numbers of presynaptic dopaminergic function for age and gender, for example, mostly independent of the equipment used and the centre performing the scan - is close to realisation. In my opinion this project has successfully shown using a single example the concept to follow and the way to go for the future. Now it needs to extend these efforts to further endorse nuclear medicine techniques.

With their strategy, EARL and EANM are in good company; many others have also realised the necessity for and benefits of standardisation and harmonisation in our field. Harmonising efforts are currently being undertaken by working groups of several societies, e.g. the QUIBA (Quantitative Imaging Biomarkers Alliance) launched by the RSNA (Radiological Society of North America), the UPICT (Uniform Protocols in Clinical Trials), a working group consisting of members of ACRIN (American College of Radiology - Imaging Network), SNM CTN (SNM Clinical Trial Network), EANM, RSNA, NIST (National Institutes of Standards), EORTC (European Organization for Research and Treatment of Cancer), NCRI (National Cancer Research Institute), QUIN (Quantitative Imaging Network) set up by the National Cancer Institute, the SNM Clinical Trial Network and others. These widespread multidisciplinary attempts will strengthen the recognition of nuclear medicine procedures, both in clinical practice and clinical trials.

Currently EARL is extending its activities to an FDG-PET/ $\mathrm{CT}$ accreditation programme (inter alia in collaboration with EORTC). The aim of this project is to provide a minimum standard of PET/CT scanner performance in order to harmonise acquisition and interpretation of PET/CT scans. With the suggested and accredited procedures a department will be able to compare, exchange and combine FDG-PET/CT findings, which is important both for eligibility as a participant in multicentre studies and for routine patient examinations. Details of this programme can readily be found at the EANM/EARL website (http://earl.eanm.org). I am convinced that this type of effort will boost the credibility of nuclear medicine procedures and is a prerequisite for further strengthening molecular imaging in Europe.

\section{References}

1. Dickson JC, Tossici-Bolt L, Sera T, de Nijs R, Booij J, Bagnara $\mathrm{MC}$, et al. Proposal for the standardisation of multi-centre trials in nuclear medicine imaging: prerequisites for a European ${ }^{123} \mathrm{I}-\mathrm{FP}-$ CIT SPECT database. Eur J Nucl Med Mol Imaging. 2011. doi:10.1007/s00259-011-1884-z.

2. Darcourt J, Booij J, Tatsch K, Varrone A, Vanderborght T, Kapucu $\mathrm{OL}$, et al. EANM procedure guidelines for brain neurotransmission SPECT using (123)I-labelled dopamine transporter ligands, version 2. Eur J Nucl Med Mol Imaging. 2010;37(2):443-50.

3. Tossici-Bolt L, Dickson JD, Sera T, de Nijs R, Bagnara MC, Jonsson $\mathrm{C}$, et al. Calibration of gamma camera systems for a multicentre European ${ }^{123}$ I-FP-CIT SPECT normal database. Eur J Nucl Med Mol Imaging. 2011;38(8):1529-40. 